\title{
Synthesis and antimicrobial activity of new triazolopyridinyl phenothiazines
}

\author{
Jignesh P. Raval* and Kishor R. Desai \\ ROFEL, Shri G. M. Bilakhia College of Applied Sciences, Vapi - 396 191India \\ Department of Chemistry, South Gujarat University, Surat - 395 007India \\ E-mail: drjpraval@hotmail.com
}

(received 19 Nov 04; accepted 21 Feb 05; published on the web 27 Feb 05)

\begin{abstract}
A variety of $N$ (2-methyl-7-aryl-8-cyano-[1,2,4] triazolo [1,5-a] pyridin-5-yl) phenothiazines 6a - d were synthesized by using chalcones of $N$ - acetylphenothiazine. And the structures of these compounds were confirmed by IR, NMR $\left({ }^{1} \mathrm{H} \&{ }^{13} \mathrm{C}\right) \&$ Mass spectral analysis. The newly synthesized compounds were also evaluated for antimicrobial activity against variety of bacterial strains and some of these compounds have shown significant antibacterial and antifungal activities.
\end{abstract}

Keywords: Chalcones, phenothiazines, triazolopyridinyl phenothiazines, IR, NMR $\left({ }^{1} \mathrm{H} \&{ }^{13} \mathrm{C}\right)$, Mass spectral analysis, Active $\mathrm{MnO}_{2}$, antibacterial and antifungal activities

\section{Introduction}

Among a wide variety of heterocycles that have been explored for developing pharmaceutically important molecules such as cyanopyridines ${ }^{1}$ and triazolopyridines ${ }^{2}$ have played an important role in medicinal chemistry. They are reported to possess a broad spectrum of biological activity such as potential cardiovascular agents ${ }^{2(c)}$ antiviral, ${ }^{3}$ CNS depressant, ${ }^{4}$ bactericidal, ${ }^{5}$ ulcer inhibitors $^{6}$ etc. Furthermore researchers have also revealed that Phenothiazine derivatives constitute an important class of compounds possessing diverse type of biological properties including antiviral, ${ }^{7}$ antiparasitic, ${ }^{8}$ antiparkinsonian, ${ }^{9}$ anticonvulsant, ${ }^{10}$ antihistaminic ${ }^{11}$ as well as anthelmintic $^{12}$ properties.

These observations led the authors to undertake the synthesis of some $N$ (2-methyl-7-aryl-8cyano[1,2,4]triazolo[1,5-a]pyridin-5-yl)phenothiazine 6a - $\mathbf{d}$ and evaluate their antimicrobial activities. 


\section{Results and Discussion}

\section{Chemistry}

Chalcones 3a-d of $\mathrm{N}$-Acetylphenothiazine were obtained from $\mathrm{N}$-acetylphenothiazine 1 using various aldehydes 2a-d. Chalcones 3a-d on treatment with malonitrile in the presence of ammonium acetate gave 4a-d. Compounds 4a-d when treated with acetonitrile in the presence of $\mathrm{AlCl}_{3}$ afforded 5a-d. Compounds 5a-d on oxidation with active $\mathrm{MnO}_{2}$ furnished 6a-d ( Scheme.1). The structures of the above compounds were in agreement with spectral and analytical data.

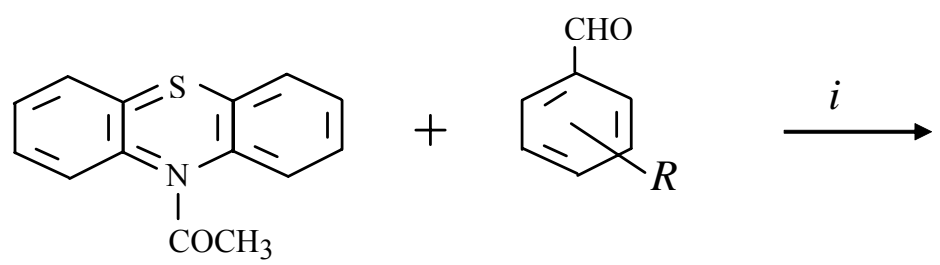

1

$2 a-d$

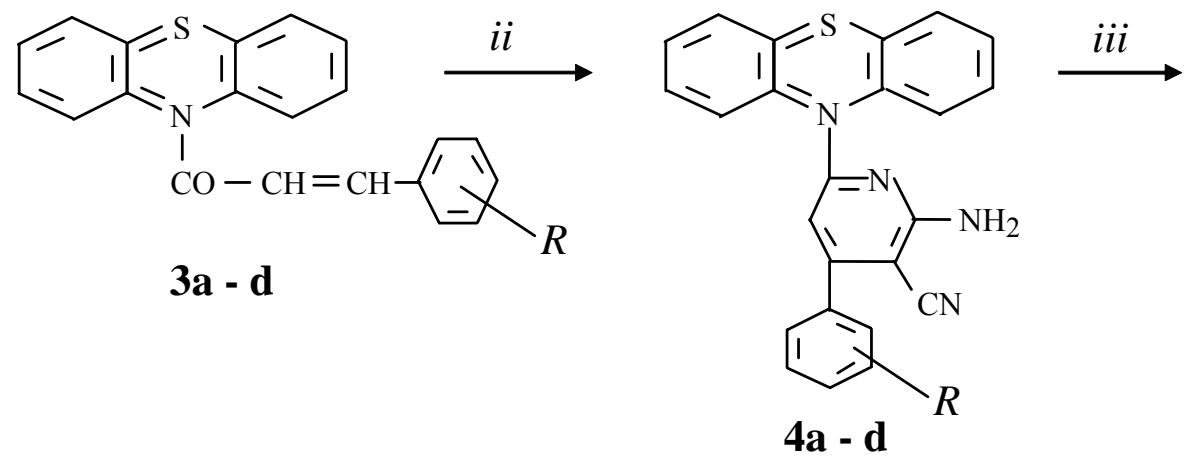

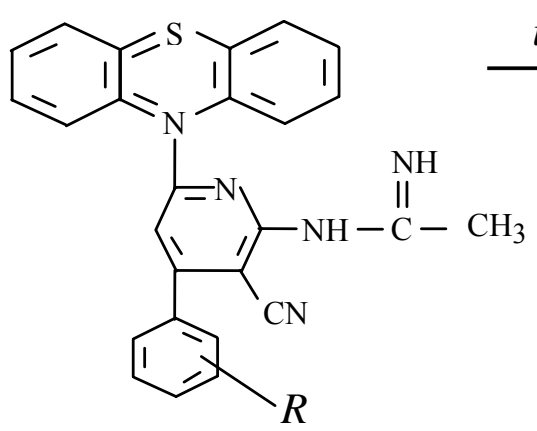

$5 a-d$<smiles>[R]c1cccc(-c2cc(N3c4ccccc4Sc4ccccc43)n3nc(C)nc3c2C#N)c1</smiles>

6a - d

Scheme 1. $\mathrm{R}=\mathrm{H}, 4-\mathrm{OCH}_{3}, 4-\mathrm{N}\left(\mathrm{CH}_{3}\right)_{2}, 4-\mathrm{OH}-3-\mathrm{OCH}_{3}$.

Reagents and reaction conditions. (i) $\mathrm{NaOH} / \mathrm{MeOH}$; (ii) $\mathrm{CH}_{2}(\mathrm{CN})_{2}, \mathrm{CH}_{3} \mathrm{COONH}_{4}$; (iii) acetonitrile, $\mathrm{AlCl}_{3}$; (iv) $\mathrm{MnO}_{2}$. 


\section{Antimicrobial activity}

The minimum inhibition concentration (MIC) was determined using the streak plate and cup plate method by measuring the zone of inhibition according to a standard procedure. ${ }^{13}$ All the newly synthesized compounds were screened in vitro for their antimicrobial activity against a variety of bacterial strains such as Staphylococcus aureus, Salmonella paratyphi, Escherichia coli, Shigella flexneri, Pseudomonas auregenosa, Bacillus subtilis, and fungi such as Cerevesae vitae, Candida albicans, Aspergillus niger ( Table.1). The MIC of the compounds was defined, as the lowest concentration at which there was $80 \%$ inhibition of growth compared with the growth for a drug free control ${ }^{14}$. Standard inhibition of zone size for Ciprofloxacin, Cloxacillin and for Gentamycin ${ }^{14}$ is $(++++)$ at $\leq 50 \mu \mathrm{gm} / \mathrm{mL}$ against all microbes.

Table 1. Antimicrobial activities of the compounds $4 \mathbf{a}-\mathbf{d}, 5 \mathbf{a}-\mathbf{d}$, and $\mathbf{6 a}-\mathbf{d}$

\begin{tabular}{cccccccccc}
\hline \multirow{2}{*}{ Compd } & \multicolumn{9}{c}{ Antibacterial activity } \\
\cline { 2 - 10 } & $S . a$ & $S . p$ & $E . c$ & $S . f$ & $P . a$ & B. $s$ & $C . a$ & $A . n$ & $C . v$ \\
\hline $\mathbf{4 a}$ & ++ & ++ & ++ & ++ & + & ++ & ++ & ++ & ++ \\
$\mathbf{4 b}$ & ++ & ++ & ++ & ++ & ++ & +++ & - & - & - \\
$\mathbf{4 c}$ & ++++ & ++ & +++ & ++ & + & - & ++ & + & + \\
$\mathbf{4 d}$ & ++ & ++ & ++ & ++ & + & ++ & + & ++ & ++ \\
$\mathbf{5 a}$ & ++++ & ++ & +++ & + & ++ & ++ & ++ & - & - \\
$\mathbf{5 b}$ & +++ & ++ & +++ & ++ & ++ & ++ & - & ++ & ++ \\
$\mathbf{5 c}$ & ++ & + & ++ & + & +++ & ++ & ++ & + & + \\
$\mathbf{5 d}$ & ++ & ++ & +++ & + & ++ & ++ & - & - & - \\
$\mathbf{6 a}$ & ++++ & ++ & +++ & + & + & ++ & ++ & + & + \\
$\mathbf{6 b}$ & ++++ & ++ & ++ & + & +++ & ++ & + & + & + \\
$\mathbf{6 c}$ & ++ & ++ & +++ & ++ & ++ & ++ & ++ & - & + \\
$\mathbf{6 d}$ & ++ & + & +++ & +++ & + & ++ & + & ++ & ++ \\
\hline
\end{tabular}

$50 \mu \mathrm{gm} / \mathrm{mL}=++++, 100 \mu \mathrm{gm} / \mathrm{mL}=+++, 150 \mu \mathrm{gm} / \mathrm{mL}=++, 200 \mu \mathrm{gm} / \mathrm{mL}=+$,

Not active upto $200 \mu \mathrm{gm} / \mathrm{mL}=-$

Ciprofloxacin, Cloxacillin \& Gentamycin ${ }^{14}$ is $(++++)$ at $\leq 50 \mu \mathrm{gm} / \mathrm{mL}$

S. $a=$ Staphylococcus aureus, S. $p=$ Salmonella paratyphi, E. $c=$ Escherichia coli,

S. $f=$ Shigella flexneri, $P . a=$ Pseudomonas auregenosa, $B . s=$ Bacillus subtilis,

C. $v=$ Cerevesae vitae, $C . a=$ Candida albicans, $A . n=$ Aspergillus niger 


\section{Conclusions}

A new series of antimicrobial agents was designed and by visualizing the antimicrobial data it could be observed that compounds of the series showed activity ranging from $50 \mu \mathrm{gm} / \mathrm{mL}$ to $200 \mu \mathrm{gm} / \mathrm{mL}$. The standard drugs used for comparision were Ciprofloxacin, Cloxacillin and Gentamycin. By visualizing the antimicrobial data it could be observed that some of the compounds possess significant activity.

\section{Experimental Section}

General Procedures. All the melting points were determined on a Cintex melting point apparatus and are uncorrected. The IR - spectra $\left(v_{\max } \mathrm{cm}^{-1}\right)$ were recorded on Perkin - Elmer 783 Spectrophotometer and NMR $\left({ }^{1} \mathrm{H}\right.$ and $\left.{ }^{13} \mathrm{C}\right)$ spectra in TFA (Chemical shifts in $\left.\delta \mathrm{ppm}\right)$ on Bruker AMX500 MHz using TMS as an internal standard, and mass spectra on Jeol D-300 spectrometer. Elemental analysis was performed on Carlo Erba - 1108 analyzer.

$N$-Acetylphenothiazine (1). ${ }^{15}$ To the solution of $10 \mathrm{H}$-phenothiazine $(0.01$ mole) in dry benzene $(50 \mathrm{~mL})$, acetyl chloride $(0.01 \mathrm{~mole})$ was added drop wise at $0-5^{\circ} \mathrm{C}$. the reaction mixture was stirred for $3-4 \mathrm{hr}$. at room temperature. After being stirred, the reaction mixture was kept overnight. The resulting mixture was distilled off and poured onto ice. The solid thus obtained was recrystallized from ethanol/ water to afford 1. yield $76 \%$, m.p. $197-98^{\circ} \mathrm{C}$ (reported ${ }^{15}$ m.p. $\left.198^{\circ} \mathrm{C}\right)$; IR $(\mathrm{KBr}) \mathrm{cm}^{-1}: 1650(\mathrm{CO}), 1450(\mathrm{C}-\mathrm{N}), 1580(\mathrm{C}=\mathrm{C}$ of aromatic ring); ${ }^{1} \mathrm{H}$ NMR $\left(\mathrm{CDCl}_{3}-\mathrm{d}_{6}\right): \delta 7.00-7.48(\mathrm{~m}, 8 \mathrm{~h}, \mathrm{Ar}-\mathrm{H}), 2.60\left(\mathrm{~s}, 3 \mathrm{H}, \mathrm{COCH}_{3}\right) ; \mathrm{MS}: \mathrm{m} / \mathrm{z}(\%)$ : 241(64\%)M $\mathrm{M}^{+}$, 266(77), 198 (100), 166 (66), 153 (34); Anal. Calcd. for $\mathrm{C}_{14} \mathrm{H}_{11} \mathrm{NOS}$ : C, 69.71; H, 4.56; N, 5.81. Found: C, 69.77; N, 4.59; N, 5.79\%.

$N$-Benzylideneacetylphenothiazine (3a). ${ }^{16}$ To the solution of $N$-acetylphenothiazine $1(0.01$ mole) in absolute methanol $(50 \mathrm{~mL})$, benzaldehyde $2 \mathrm{a}(0.01 \mathrm{~mole})$ was added on the presence of $2 \% \mathrm{NaOH}(2 \mathrm{~mL})$ and refluxed for $10-12 \mathrm{hr}$. After refluxing, the reaction mixture was concentrated to half of its volume and poured onto ice, extracted with benzene and solvent was removed to get residue, which was washed several times with water and finally recrystallized from ethanol to give 3a: yield $72 \%$, m.p. $168^{\circ} \mathrm{C}$; IR $(\mathrm{KBr}) \mathrm{cm}^{-1}: 1650(\mathrm{CO}), 1620(\mathrm{CH}=\mathrm{CH})$, $1575(\mathrm{C}=\mathrm{C}$ aromatic ring $) \mathrm{cm}-1 ;{ }^{1} \mathrm{H}$ NMR $\left(\mathrm{CDCl}_{3}-\mathrm{d}_{6}\right): \delta 8.61(\mathrm{~d}, 1 \mathrm{H},=\mathrm{CH}-\mathrm{Ar}), 7.00-7.67$ $(\mathrm{m}, 12 \mathrm{H}, \mathrm{Ar}-\mathrm{H}), 6.71(\mathrm{~d}, 1 \mathrm{H}, \mathrm{COCH}=) ; \mathrm{MS}: \mathrm{m} / \mathrm{z}(\%): 329(88.5) \mathrm{M}^{+}, 266$ (72), 198 (100), 166 (63), 153 (31 ),103 (65), 90 (77), 77 (72).

Similarly, other chalcone derivatives $3 \mathbf{b}-\mathbf{d}$ were synthesized by condensing $N-$ Acetylphenothiazine with various aldehydes $\mathbf{2 b}-\mathbf{d}$ according to method reported in the literature. $^{16}$ 
General procedure for (4a-d) $\mathbf{N}$-(2-Amino-4-phenyl-3-cyanopyridin-6-yl)phenothiazine ${ }^{17}$ 4a A mixture of chalcones $3 \mathbf{a}-\mathbf{d}(0.01$ mole $)$, malonitrile $(0.01$ mole, $0.66 \mathrm{~g})$ and ammonium acetate $(0.08$ mole, $6.16 \mathrm{~g})$ dissolved in ethanol $(20 \mathrm{~mL})$ was refluxed on a water bath for $6 \mathrm{hr}$. It was then decomposed in crushed ice, neutralized with dil. $\mathrm{HCl}$ and recrystallized from methanol to give product (4a). m.p. $197^{\circ} \mathrm{C} ;{ }^{1} \mathrm{H}$ NMR (DMSO $-\mathrm{d}_{6}$ ): $\delta 8.41$ (s, 2H, $\mathrm{NH}_{2}, \mathrm{D}_{2} \mathrm{O}$ exchangeable), $7.00-8.00(\mathrm{~m}, 13 \mathrm{H}, \mathrm{Ar}-\mathrm{H}) .{ }^{13} \mathrm{C}$ NMR : $118.6(-\mathrm{CN}), 120-140(\mathrm{Ar}-\mathrm{C})$, $159.9\left(\mathrm{C}_{5},>\mathrm{C}=\mathrm{N}\right), 178.2\left(\mathrm{C}_{2}, \mathrm{~N}-\mathrm{C}-\mathrm{N}\right)$; MS : m/z (\%) : 392 (72) $\mathrm{M}^{+}, 350$ (3.4), 301 (32.6), 286 (33.4), 198 (100), 166 (65), 153 (35), 108 (30), 77 (33.7); Anal. Calcd. for $\mathrm{C}_{24} \mathrm{H}_{16} \mathrm{~N}_{4} \mathrm{~S}$ : C, 73.46; H, 4.08; N, 14.28. Found: C, 73.51; H, 4.09; N, 14.26\%.

$\mathrm{N}$-(2-Amino-4-(4-methoxyphenyl)-3-cyanopyridin-6-yl )phenothiazine (4b). M.p. $213{ }^{\circ} \mathrm{C} ;{ }^{1} \mathrm{H}$ NMR (DMSO - $\left.\mathrm{d}_{6}\right): \delta 3.72\left(\mathrm{~s}, 3 \mathrm{H}, \mathrm{OCH}_{3}\right),, 8.19\left(\mathrm{~s}, 2 \mathrm{H}, \mathrm{NH}_{2}, \mathrm{D}_{2} \mathrm{O}\right.$ exchangeable), $7.00-8.10$ $(\mathrm{m}, 12 \mathrm{H}, \mathrm{Ar}-\mathrm{H}) .{ }^{13} \mathrm{C} \mathrm{NMR}: 55.6\left(\mathrm{OCH}_{3}\right), 114.6(-\mathrm{CN}), 125-140(\mathrm{Ar}-\mathrm{C}), 157.9\left(\mathrm{C}_{5},>\mathrm{C}=\right.$ $\mathrm{N}), 179.1\left(\mathrm{C}_{2}, \mathrm{~N}-\mathrm{C}-\mathrm{N}\right)$; MS : m/z (\%) : 422 (62) $\mathrm{M}^{+}, 394$ (5.5), 391 (31.6), 345 (77.0), 331 (12.7), 199 (95), 165 (35), 153 (30), 78 (32.7); Anal. Calcd. for $\mathrm{C}_{25} \mathrm{H}_{18} \mathrm{ON}_{4} \mathrm{~S}: \mathrm{C}, 71.09$; H, 4.26; O, 3.79; N, 13.27. Found: C, 71.01; H, 4.29;N, 3.77; N, 13.29\%.

$\boldsymbol{N}$-(2-Amino-4-(4- $\mathbf{N}, \boldsymbol{N}$-dimethylaminophenyl)-3-cyanopyridin-6-yl)phenothiazine (4c). M.p. $237{ }^{\circ} \mathrm{C} ;{ }^{1} \mathrm{H}$ NMR (DMSO - $\left.\mathrm{d}_{6}\right): \delta 3.11\left[\mathrm{~s}, 6 \mathrm{H}, \mathrm{N}\left(\mathrm{CH}_{3}\right)_{2}\right], 8.49$ (s, 2H, $\mathrm{NH}_{2}, \mathrm{D}_{2} \mathrm{O}$ exchangeable), $7.00-8.10(\mathrm{~m}, 12 \mathrm{H}, \mathrm{Ar}-\mathrm{H}) .{ }^{13} \mathrm{C} \mathrm{NMR}: 40.3\left[\mathrm{~N}\left(\mathrm{CH}_{3}\right)_{2}\right], 59.6\left(\mathrm{OCH}_{3}\right), 115.9(-\mathrm{CN}), 130-140$ $(\mathrm{Ar}-\mathrm{C}), 152.9\left(\mathrm{C}_{5},>\mathrm{C}=\mathrm{N}\right), 178.9\left(\mathrm{C}_{2}, \mathrm{~N}-\mathrm{C}-\mathrm{N}\right) ; \mathrm{MS}: \mathrm{m} / \mathrm{z}(\%): 435(72) \mathrm{M}^{+}, 420(7.4), 407$ (42.1), 390 (33.4), 358 (47.7), 343 (65), 199 (100), 166 (30),153 (42.6), 108 (19.7), 78 (33.5); Anal. Calcd. for $\mathrm{C}_{26} \mathrm{H}_{21} \mathrm{~N}_{5} \mathrm{~S}$ : C, 71.72; H, 4.85; N, 16.09. Found: C, 71.77; H, 4.89; N, 16.07\%.

$\mathrm{N}$-(2-Amino-4-(3-hydroxy-4-methoxyphenyl)-3-cyanopyridin-6-yl)phenothiazine (4d). M.p. $254{ }^{\circ} \mathrm{C} ;{ }^{1} \mathrm{H}$ NMR (DMSO - $\left.\mathrm{d}_{6}\right): \delta 3.77\left(\mathrm{~s}, 3 \mathrm{H}, \mathrm{OCH}_{3}\right) ; 8.46\left(\mathrm{~s}, 2 \mathrm{H}, \mathrm{NH}_{2}, \mathrm{D}_{2} \mathrm{O}\right.$ exchangeable), $7.00-8.00(\mathrm{~m}, 11 \mathrm{H}, \mathrm{Ar}-\mathrm{H}), 10.77\left(\mathrm{~s}, 1 \mathrm{H}, \mathrm{OH}, \mathrm{D}_{2} \mathrm{O}\right.$ exchangeable); ${ }^{13} \mathrm{C} \mathrm{NMR}: 59.6\left(\mathrm{OCH}_{3}\right)$, $111.6(-\mathrm{CN}), 120-150(\mathrm{Ar}-\mathrm{C}), 160.9\left(\mathrm{C}_{5},>\mathrm{C}=\mathrm{N}\right), 162.0(\mathrm{C}-\mathrm{OH}), 179.9\left(\mathrm{C}_{2}, \mathrm{~N}-\mathrm{C}-\mathrm{N}\right)$; MS : m/z (\%) : 438 (72) $\mathrm{M}^{+}, 421$ (4.4), 410 (22.6), 407 (23.4), 361 (45.6), 346 (65.7), 198 (100), 166 (75), 154 (45), 108 (50), 77 (31.7); Anal. Calcd. for $\mathrm{C}_{25} \mathrm{H}_{18} \mathrm{O}_{2} \mathrm{~N}_{4} \mathrm{~S}$ : C, 68.49; H, 4.10; O, 7.30; N, 12.78. Found: C, 68.41; H, 4.11; O, 7.28; N, 12.79\%.

\section{General procedure for $(5 \mathrm{a}-\mathrm{d}) \mathrm{N}$-(2-Acetamidino-4-phenyl-3-cyanopyridin-6-yl)phenothiazine $5 \mathrm{a}$} A mixture $3 \mathbf{a}-\mathbf{d}\left(0.01\right.$ mole), powdered anhydrous $\mathrm{AlCl}_{3}$ and acetonitrile $(1.0 \mathrm{~mL})$ was heated on an oil - bath at $150-160{ }^{\circ} \mathrm{C}$ for $8 \mathrm{hr}$. The contents were cooled and decomposed in ice - cold $\mathrm{HCl}$. The product obtained was filtered, washed with water and recrystallized from ethanol to give 5a. m.p. $207{ }^{\circ} \mathrm{C} ;{ }^{1} \mathrm{H}$ NMR (DMSO $\left.-\mathrm{d}_{6}\right): \delta 2.51\left(\mathrm{~s}, 3 \mathrm{H}, \mathrm{CH}_{3}\right) ; 4.48\left(\mathrm{~s}, 1 \mathrm{H}, \mathrm{NH}, \mathrm{D}_{2} \mathrm{O}\right.$ exchangeable), $7.10-8.20(\mathrm{~m}, 13 \mathrm{H}, \mathrm{Ar}-\mathrm{H}) .{ }^{13} \mathrm{C}$ NMR: $34.9\left(\mathrm{CH}_{3}\right), 115.9(-\mathrm{CN}), 130-140$ $(\mathrm{Ar}-\mathrm{C}), 151.1\left(\mathrm{C}_{6},>\mathrm{C}=\mathrm{N}\right), 175.7\left(\mathrm{C}_{2}, \mathrm{~N}-\mathrm{C}=\mathrm{N}\right), 180.0\left(\mathrm{C}_{2}, \mathrm{~N}-\mathrm{C}=\mathrm{N}\right): \mathrm{MS}: \mathrm{m} / \mathrm{z}(\%)$ : 433 (32) $\mathrm{M}^{+}, 418$ (54), 405 (23), 376 (15.3), 356 (27), 331 (29.2), 263 (57.7), 199 (99), 167 (62), 153 (59.2), 108 (34), 78 (6.8); Anal. Calcd. for $\mathrm{C}_{26} \mathrm{H}_{19} \mathrm{~N}_{5} \mathrm{~S}$ : C, 72.05; H, 4.38; N, 16.16. Found: C, 72.01; H, 4.33; N, 16.19\%. 
$\mathbf{N}$-(2-Acetamidino-4-(4-methoxyphenyl)-3-cyanopyridin-6-yl)phenothiazine(5b). M.p. $187{ }^{\circ} \mathrm{C} ;{ }^{1} \mathrm{H}$ NMR (DMSO - $\left.\mathrm{d}_{6}\right): \delta 2.57\left(\mathrm{~s}, 3 \mathrm{H}, \mathrm{CH}_{3}\right) ; 3.85\left(\mathrm{~s}, 3 \mathrm{H}, \mathrm{OCH}_{3}\right), 4.46\left(\mathrm{~s}, 1 \mathrm{H}, \mathrm{NH}, \mathrm{D}_{2} \mathrm{O}\right.$ exchangeable), $7.10-8.10(\mathrm{~m}, 12 \mathrm{H}, \mathrm{Ar}-\mathrm{H}) .{ }^{13} \mathrm{C} \mathrm{NMR}: 33.9\left(\mathrm{CH}_{3}\right), 58.9\left(\mathrm{OCH}_{3}\right), 115.2(-\mathrm{CN})$, $130-140(\mathrm{Ar}-\mathrm{C}), 152.5\left(\mathrm{C}_{6},>\mathrm{C}=\mathrm{N}\right), 174.9\left(\mathrm{C}_{2}, \mathrm{~N}-\mathrm{C}=\mathrm{N}\right), 181.0\left(\mathrm{C}_{2}{ }^{\prime}, \mathrm{N}-\mathrm{C}=\mathrm{N}\right)$ : $\mathrm{MS}$ : m/z (\%) : 463 (35) M+ , 448 (51), 432 (47.7), 406 (25), 386 (25.2), 361 (26), 293 (39.1), 199 (100), 165 (72), 152 (57.1), 166 (62), 76 (7.8); Anal. Calcd. for $\mathrm{C}_{27} \mathrm{H}_{21} \mathrm{ON} \mathrm{ON}_{5} \mathrm{~S}$ : C, 69.97; H, 4.53; O, 3.45; N, 15.11. Found: C, 69.92; H, 4.52; O, 3.42; N, 15.19\%.

$\mathbf{N}$-(2-Acetamidino-4-(4- $\mathbf{N , N}$-dimethylaminophenyl)-3-cyanopyridin-6-yl)phenothiazine(5c). M.p. $218^{\circ} \mathrm{C} ;{ }^{1} \mathrm{H}$ NMR (DMSO - $\left.\mathrm{d}_{6}\right): \delta 2.46\left[\mathrm{~s}, 6 \mathrm{H}, \mathrm{N}\left(\mathrm{CH}_{3}\right)_{2}\right], 2.59$ (s, 3H, $\left.\mathrm{CH}_{3}\right) ; 3.85(\mathrm{~s}, 3 \mathrm{H}$, $\left.\mathrm{OCH}_{3}\right), 4.44\left(\mathrm{~s}, 1 \mathrm{H}, \mathrm{NH}, \mathrm{D}_{2} \mathrm{O}\right.$ exchangeable), $7.10-8.20(\mathrm{~m}, 12 \mathrm{H}, \mathrm{Ar}-\mathrm{H}) .{ }^{13} \mathrm{C} \mathrm{NMR}: 34.2$ $\left(\mathrm{CH}_{3}\right), 49.3\left[\left(\mathrm{~N}-\left(\mathrm{CH}_{3}\right)_{2}\right], 114.9(-\mathrm{CN}), 130-140(\mathrm{Ar}-\mathrm{C}), 154.1\left(\mathrm{C}_{6},>\mathrm{C}=\mathrm{N}\right), 171.7\left(\mathrm{C}_{2}, \mathrm{~N}-\right.\right.$ $\mathrm{C}=\mathrm{N}), 182.1\left(\mathrm{C}_{2}, \mathrm{~N}-\mathrm{C}=\mathrm{N}\right): \mathrm{MS}: \mathrm{m} / \mathrm{z}(\%): 477(52) \mathrm{M}^{+}, 462$ (54), 446 (19.7), 420 (21), 400 (13.5), 375 (10.1), 306 (26), 198 (99), 166 (65), 153(69.2), 106 (31), 76(6.9).

$\mathbf{N}$ (2-Acetamidino-4-(3-hydroxy-4-methoxyphenyl)-3-cyanopyridin-6-yl)phenothiazine (5d). M.p. $198^{\circ} \mathrm{C} ;{ }^{1} \mathrm{H}$ NMR (DMSO - d $)$ ) $\delta 3.61(\mathrm{dd}, 1 \mathrm{H}, \mathrm{OH}), 3.82\left(\mathrm{~s}, 3 \mathrm{H}, \mathrm{OCH}_{3}\right), 2.57\left(\mathrm{~s}, 3 \mathrm{H}, \mathrm{CH}_{3}\right)$; $3.85\left(\mathrm{~s}, 3 \mathrm{H}, \mathrm{OCH}_{3}\right), 4.49\left(\mathrm{~s}, 1 \mathrm{H}, \mathrm{NH}, \mathrm{D}_{2} \mathrm{O}\right.$ exchangeable $), 7.10-8.10(\mathrm{~m}, 11 \mathrm{H}, \mathrm{Ar}-\mathrm{H}) .{ }^{13} \mathrm{C}$ NMR : $35.1\left(\mathrm{CH}_{3}\right), 58.7\left(\mathrm{OCH}_{3}\right), 115.7(-\mathrm{CN}), 159.1(\mathrm{C}-\mathrm{OH}), 130-140(\mathrm{Ar}-\mathrm{C}), 152.3\left(\mathrm{C}_{6}\right.$, $>\mathrm{C}=\mathrm{N}), 171.9\left(\mathrm{C}_{2}, \mathrm{~N}-\mathrm{C}=\mathrm{N}\right), 181.9\left(\mathrm{C}_{2}{ }^{\prime}, \mathrm{N}-\mathrm{C}=\mathrm{N}\right) ; \mathrm{MS}: \mathrm{m} / \mathrm{z}(\%): 479(41) \mathrm{M}^{+}, 464(64), 448$ (29.2), 422 (24), 402 (14.2), 309 (37), 198 (99.2), 166 (62.7), 152 (59.7), 108 (34), 76 (6.9); Anal. Calcd. for $\mathrm{C}_{28} \mathrm{H}_{25} \mathrm{~N}_{6} \mathrm{~S}$ : C, 70.44; H, 5.24; N, 17.61. Found: C, 70.42; H, 5.21; N, 17.67\%.

Active $\mathrm{MnO}_{2}$. A solution of Manganese (II) sulphate tetrahydrate $\left.\left(\mathrm{MnSO}_{4} \cdot 4 \mathrm{H}_{2} \mathrm{O}\right) 223 \mathrm{~g}, 1 \mathrm{~mole}\right)$ in $300 \mathrm{~mL}$ of water and $240 \mathrm{~mL}$ (2.5 moles) of $40 \%$ aqueous $\mathrm{NaOH}$ solution was added simultaneously during $1 \mathrm{hr}$ to a hot stirred solution of $190 \mathrm{~g}$ of $\mathrm{KmnO}_{4}$ in $1200 \mathrm{~mL}$ of water. The stirring was continued for further $1 \mathrm{hr}$ and the brown precipitate of $\mathrm{MnO}_{2}$ was filtered and washed with water until the washings were colourless. The precipitate was dried in oven at $100-$ $120^{\circ} \mathrm{C}$ and grinded to fine powder.

General procedure for $(6 a-$ d) $N$-(2-Methyl-7-phenyl-8-cyano-[1,2,4]triazolo[1,5-a]pyridin5-yl)phenothiazine 6a.

A mixture of $5 \mathbf{a}(0.01$ mole $)$ and active $\mathrm{MnO}_{2}(4.0 \mathrm{~g})$ was taken in benzene $(50 \mathrm{~mL})$. The contents were refluxed for $11 \mathrm{hr}$. and filtered hot. Solvent was removed to give the product. It was filtered, washed and recrystallized from alcohol to give product. 6a. m.p. $231^{\circ} \mathrm{C}$; ${ }^{1} \mathrm{H}$ NMR $\left(\mathrm{DMSO}-\mathrm{d}_{6}\right): \delta 2.55\left(\mathrm{~s}, 3 \mathrm{H}, \mathrm{CH}_{3}\right), 7.00-8.00(\mathrm{~m}, 13 \mathrm{H}, \mathrm{Ar}-\mathrm{H}) .{ }^{13} \mathrm{C} \mathrm{NMR}: 32.2\left(\mathrm{CH}_{3}\right), 115.4(-$ $\mathrm{CN}), 125-140(\mathrm{Ar}-\mathrm{C}), 150.7\left(\mathrm{C}_{8},>\mathrm{C}=\mathrm{N}\right), 174.4(\mathrm{~N}-\mathrm{C}=\mathrm{N}), 182\left(\mathrm{C}_{3}, \mathrm{~N}-\mathrm{C}=\mathrm{N}\right) ; \mathrm{MS}: \mathrm{m} / \mathrm{z}$ (\%) : 431 (20) M+, 372 (3.5), 340 (3.5), 312 (15.2), 302 (16.5), 199 (99.1), 166 (31.2), 152 (32.2), 106 (23.5), 77 (32.6); Anal. Calcd. for $\mathrm{C}_{26} \mathrm{H}_{17} \mathrm{~N}_{5} \mathrm{~S}$ : C, 72.38; H, 3.94; N, 16.24. Found: C, 72.39; H, 3.95; N, 16.27\%.

N-(2-Methyl-7-(4-methoxyphenyl)-8-cyano-[1,2,4]triazolo[1,5-a]pyridin-5-yl)phenothiazine (6b). M.p. $224^{\circ} \mathrm{C} ;{ }^{1} \mathrm{H}$ NMR (DMSO - $\left.\mathrm{d}_{6}\right): \delta 2.51\left(\mathrm{~s}, 3 \mathrm{H}, \mathrm{CH}_{3}\right), 3.35\left(\mathrm{~s}, 3 \mathrm{H}, \mathrm{OCH}_{3}\right), 7.00-8.00$ $(\mathrm{m}, 12 \mathrm{H}, \mathrm{Ar}-\mathrm{H}) .{ }^{13} \mathrm{C}$ NMR: $32.5\left(\mathrm{CH}_{3}\right), 114.7(-\mathrm{CN}), 125-140(\mathrm{Ar}-\mathrm{C}), 149.1\left(\mathrm{C}_{8},>\mathrm{C}=\mathrm{N}\right)$, 
$154.8\left(\mathrm{C}_{4}{ }^{\prime}, \mathrm{C}-\mathrm{OCH}_{3}\right), 175.2(\mathrm{~N}-\mathrm{C}=\mathrm{N}), 184\left(\mathrm{C}_{3}, \mathrm{~N}-\mathrm{C}=\mathrm{N}\right) ; \mathrm{MS}: \mathrm{m} / \mathrm{z}(\%): 461(27) \mathrm{M}^{+}$, 444 (15.2), 430 (16.5), 402 (4.5), 370 (4.8), 332 (8.5), 199 (100), 163 (31.2), 153 (25.9), 106 (31.7), 77 (30.6); Anal. Calcd. for $\mathrm{C}_{27} \mathrm{H}_{19} \mathrm{ON}_{5} \mathrm{~S}$ : C, 70.28; H, 4.12; O, 3.47; N, 15.18. Found: C, 70.29; H, 4.15; O, 3.45; N, 15.19\%.

$\boldsymbol{N}$-(2-Methyl-7-(4- $\mathbf{N , N - d i m e t h y l a m i n o p h e n y l ) - 8 - c y a n o - [ 1 , 2 , 4 ] t r a z o l o [ 1 , 5 - a ] p y r i d i n - 5 - y l ) ~}$ phenothiazine (6c). M.p. $213^{\circ} \mathrm{C} ;{ }^{1} \mathrm{H}$ NMR (DMSO - $\left.\mathrm{d}_{6}\right): \delta 2.46\left[\mathrm{~s}, 6 \mathrm{H}, \mathrm{N}\left(\mathrm{CH}_{3}\right)_{2}\right], 2.59(\mathrm{~s}, 3 \mathrm{H}$, $\left.\mathrm{CH}_{3}\right), 7.00-8.00(\mathrm{~m}, 11 \mathrm{H}, \mathrm{Ar}-\mathrm{H}) .{ }^{13} \mathrm{C} \mathrm{NMR}: 32.7\left(\mathrm{CH}_{3}\right), 114.9(-\mathrm{CN}), 125-140(\mathrm{Ar}-\mathrm{C})$, $150.1\left(\mathrm{C}_{8},>\mathrm{C}=\mathrm{N}\right), 175.1(\mathrm{~N}-\mathrm{C}=\mathrm{N}), 185\left(\mathrm{C}_{3}, \mathrm{~N}-\mathrm{C}=\mathrm{N}\right) ; \mathrm{MS}: \mathrm{m} / \mathrm{z}(\%): 474(29) \mathrm{M}^{+}, 457$ (25.1), 443 (26.1), 383 (3.9), 355 (3.6), 345 (4.6), 199 (99.9), 164 (29.2), 152 (32.2), 106 (31.8), 78 (31.6); Anal. Calcd. for $\mathrm{C}_{28} \mathrm{H}_{22} \mathrm{~N}_{6} \mathrm{~S}$ : C, 70.88; H, 4.64; N, 17.72. Found: C, 70.87; H, 4.68; N, $17.77 \%$.

$\mathbf{N}$-(2-Methyl-7-(3-hydroxy-4-methoxyphenyl)-8-cyano-[1,2,4]trazolo[1,5-a]pyridin-5-yl) phenothiazine (6d). M.p. $221^{\circ} \mathrm{C} ;{ }^{1} \mathrm{H}$ NMR (DMSO - $\left.\mathrm{d}_{6}\right): \delta 2.57\left(\mathrm{~s}, 3 \mathrm{H}, \mathrm{CH}_{3}\right), 3.39(\mathrm{~s}, 3 \mathrm{H}$, $\left.\mathrm{OCH}_{3}\right), 7.00-8.00(\mathrm{~m}, 11 \mathrm{H}, \mathrm{Ar}-\mathrm{H}), 11.09\left(\mathrm{~s}, 1 \mathrm{H}, \mathrm{OH}, \mathrm{D}_{2} \mathrm{O}\right.$ exchangeable); ${ }^{13} \mathrm{C}$ NMR: 32.9 $\left(\mathrm{CH}_{3}\right), 115.1(-\mathrm{CN}), 125-140(\mathrm{Ar}-\mathrm{C}), 149.9\left(\mathrm{C}_{8},>\mathrm{C}=\mathrm{N}\right), 151.8\left(\mathrm{C}_{4}{ }^{\prime}, \mathrm{C}-\mathrm{OCH}_{3}\right), 175.7(\mathrm{~N}-$ $\mathrm{C}=\mathrm{N}), 182\left(\mathrm{C}_{3}, \mathrm{~N}-\mathrm{C}=\mathrm{N}\right)$; MS : m/z (\%) : 477 (30) $\mathrm{M}^{+}, 418$ (3.8), 358 (3.1), 386 (5.7), 348 (16.9), 460 (26.9), 446 (27.9), 199 (100), 162 (31.9), 152 (25.9), 106 (33.7), 77 (32.9); Anal. Calcd. for $\mathrm{C}_{27} \mathrm{H}_{19} \mathrm{O}_{2} \mathrm{~N}_{5} \mathrm{~S}$ : C, 67.92; H, 3.98; O, 6.70; N, 14.67. Found: C, 67.95; H, 3.92; O, 6.71; $\mathrm{N}, 14.77 \%$.

\section{Acknowledgements}

One of the authors (JPR) is thankful to (The Principal) ROFEL, Shri G. M. Bilakhia College of Applied Sciences, Vapi for providing research facilities, ROFEL, Shri G. M. Bilakhia College of Pharmacy for screening the newly synthesized compounds for their Antimicrobial activities and Mr. Priyakant R. Raval of Cyanamid India Limited, Atul for providing Spectral facilities.

\section{References}

1. (a) Klimešová, V.; Otčenášek, M.; Waisser K. Eur. J. Med. Chem. 1996, 31, 389. (b) Suloeva, E.; Yure, M.; Gudriniece, E.; Petrova, M.; Gutcaits, A. Chemistry of Heterocyclic Compounds; 2001, Vol. 37, p 872. (c) Quintela, J. M.; Peinador, C.; Botana, L.; Estevez, M.; Riguera, R. Bioorg. Med. Chem. 1997, 5, 1543.

2. (a) Abarca, B.; Ballesteros, R.; Elmasnaouy, M. Tetrahedron 1999, 55, 12881. (b) Abarca, B.; Ballesteros, R.; Elmasnaouy, M. ARKIVOC 2002, (vi), 146. (c) Abarca, B, Ballesteros, R., Elmasnaouy, M.; D’Ocón, P.; Ivorra, M. D.; Valiente, M. ARKIVOC 2002, (x), 9. (d) Abarca, B.; Ballesteros, R.; Chadlaoui, M. ARKIVOC 2002, (x), 52. (e) Abarca, B.; Ballesteros, R.; Chadlaoui, M. ARKIVOC 2004, (iv), 42. 
3. Sanghavi, Y. S.; Larson, S. B.; Robinse, R. K.; Revenkar, G. R. J Med. Chem. 1989, 32, 945.

4. Paller, M.; Ponzio, K. Chem. Abstr. 1983, 99, 158406r.

5. Prakash, L.; Sharma, R.; Shukla, S.; Goval, R. D.; Pharmazie 1993, 48, 221; Chem. Abstr. 1993, 119, 95456t.

6. Heichachiro, A.; Shinozaki, K.; Niwa, S.; Tomosho, A.; Masao, S.; Mitsui, S.; Shiggeru, U. Chem. Abstr. 1989, 110, 23891v.

7. Ddhlbom, R.; Ekstramd, T. Arch. Intern. Pharmacodynamics 1996, 159, 70.

8. Weil, C. S. Biometrics. 1952, 8, 249.

9. Harpen, B. N. J Am. Med. Assoc. 1945, 129, 1219.

10. Genzer, J. D.; Lewis, M. N.; McMillan, F. H.; King, J. A. J. Am. Chem. Soc. 1953, 75, 2206.

11. Dushay, L. J. Rev. Can. Biol. 1961, 20, 321.

12. Douglass, J. R.; Baker, N. F.; Longwest, M. W. Am. J Vet. Res. 1956, 17, 318.

13. Gradwol's Clinical Laboratory methods and diagnosis. 1970 , Vol. II, $7^{\text {th }}$ Edn; Mosby, C. V. Company: Germany, 1407.

14. National Committee for Clinical Laboratory Standards.1997, Reference method for broth dilution antifungal susceptibility testing of yeast. Approved standard NCCLS document M27 - A. (ISBN 1 - 56238 - 328 - 0, ISSN 0273 - 3099). National Committee for Clinical Laboratory Standards, 940, West Valley Road, Suite 1400, Wayne, Pennsylvania, 19807.

15. Germaine, C.; Andre, C. Chem. Abstr. 1954, 48, $2715 \mathrm{~b}$.

16. Bajaj, Kiran; Srivastava, V. K.; Kumar, Ashok. Indian J. Chem. 2004, 43B, 157.

17. Desai, J. M.; Shah, V. H. Indian J. Chem. 2003, 42B, 2595. 